\title{
Correction: How Can Artificial Intelligence Make Medicine More Preemptive?
}

\author{
Usman Iqbal $^{1,2}$, PharmD, MBA, PhD; Leo Anthony Celi ${ }^{3,4,5}$, MD, MSc; Yu-Chuan Jack Li ${ }^{2,6,7,8}$, MD, PhD \\ ${ }_{1}^{1}$ Master Program in Global Health \& Development, PhD Program in Global Health \& Health Security, College of Public Health, Taipei Medical \\ University, Taipei, Taiwan \\ ${ }^{2}$ International Center for Health Information Technology, Taipei Medical University, Taipei, Taiwan \\ ${ }^{3}$ Laboratory for Computational Physiology, Massachusetts Institute of Technology, Cambridge, MA, United States \\ ${ }^{4}$ Division of Pulmonary, Critical Care and Sleep Medicine, Beth Israel Deaconess Medical Center, Boston, MA, United States \\ ${ }^{5}$ Department of Biostatistics, Harvard TH Chan School of Public Health, Harvard University, Boston, MA, United States \\ ${ }^{6}$ Graduate Institute of Biomedical Informatics, College of Medical Science and Technology, Taipei Medical University, Taipei, Taiwan \\ ${ }^{7}$ Department of Dermatology, Taipei Municipal Wan-Fang Hospital, Taipei, Taiwan \\ ${ }^{8}$ International Medical Informatics Association, Geneva, Switzerland
}

\section{Corresponding Author:}

Yu-Chuan Jack Li, MD, PhD

Graduate Institute of Biomedical Informatics

College of Medical Science and Technology

Taipei Medical University

No 172-1, Sec 2, Keelung Rd

Daan District

Taipei, 10675

Taiwan

Phone: 88666382736 ext 7601

Email: jack@tmu.edu.tw

\section{Related Article:}

Correction of: https://www.jmir.org/2020/8/e17211

(J Med Internet Res 2020;22(8):e23645) doi: 10.2196/23645

In "How Can Artificial Intelligence Make Medicine More Preemptive?" (J Med Internet Res 2020;22(8):e17211) the authors noted one error.

The first affiliation for author Usman Iqbal originally read:

College of Public Health, Taipei Medical University, Taipei, Taiwan

This was incomplete, and has been updated to read:
Master Program in Global Health \& Development, PhD Program in Global Health \& Health Security, College of Public Health, Taipei Medical University, Taipei, Taiwan

The correction will appear in the online version of the paper on the JMIR Publications website on August 26, 2020, together with the publication of this correction notice. Because this was made after submission to PubMed, PubMed Central, and other full-text repositories, the corrected article has also been resubmitted to those repositories

This is a non-peer-reviewed article. Submitted 18.08.20; accepted 18.08.20; published 26.08.20.

Please cite as:

Iqbal U, Celi LA, Li YCJ

Correction: How Can Artificial Intelligence Make Medicine More Preemptive?

$J$ Med Internet Res 2020;22(8):e23645

URL: http://www.jmir.org/2020/8/e23645/

doi: $10.2196 / 23645$

PMID: $\underline{32845851}$ 
(CUsman Iqbal, Leo Anthony Celi, Yu-Chuan Jack Li. Originally published in the Journal of Medical Internet Research (http://www.jmir.org), 26.08.2020. This is an open-access article distributed under the terms of the Creative Commons Attribution License (https://creativecommons.org/licenses/by/4.0/), which permits unrestricted use, distribution, and reproduction in any medium, provided the original work, first published in the Journal of Medical Internet Research, is properly cited. The complete bibliographic information, a link to the original publication on http://www.jmir.org/, as well as this copyright and license information must be included. 